\title{
The Effect of Pulsatile Flow on the Peristaltic Transport of a Newtonian Fluid in a Cylindrical Tube with Permeable Wall
}

\author{
G.Sivaiah $^{1}$, S.Sreenadh ${ }^{2}$ and K.Chakradhar ${ }^{2}$ \\ ${ }^{l}$ Department of Mathematics, Govt.Degree College Rajampet-516115, Kadapa Dt. A.P. (INDIA). \\ ${ }^{2}$ Professor, Department of Mathematics, S.V. University,Tirupati-517502, Chittoor Dt. A.P. (INDIA).
}

\begin{abstract}
Peristaltic pumping is a form of the fluid transport in a flexible tube caused by a progressive wave of contraction or expansion from a region of lower pressure to higher pressure. Peristalsis is one of the major mechanisms for fluid in many biological systems. It is an automatic and vital process that moves food through the digestive tract, urine from the kidneys through the ureters into the bladder, and bile from the gall bladder into the duodenum and transport of blood through the artery with mild stenosis. In addition, the mechanism of peristalsis is applied in the design of biomechanical instruments in chas heart-lung machine. In many biomechanical devices such as small blood vessels, the tube wall is not rigid. The tissne region of the blood vessel is modelled as a permeable layer. In view of this it will interesting to study peristaltic pulsatile flow of a viscous fluid in a tube with permeable boundary. In this paper, we study the effect of the pulsatile flow on the peristaltic pumping of a Newtonian fluid in an axi symmetric cylindrical tube with permeable wall is investigated The analytical solution of this problem is given as on asymptotic expansion in the Womersly number $\beta$ which characterize the unsteadyness effect in the wave frame. The pumping characteristics are discussed.
\end{abstract}

Keywords: Pulsatile Flow, Newtonian Fluid, Reynolds Number.

\section{Introduction}

Peristaltic pumping is a form of the fluid transport in a flexible tube caused by a progressive wave of contraction or expansion from a region of lower pressure to higher pressure. Peristalsis is one of the major mechanisms for fluid in many biological systems. It is an automatic and vital process that moves food through the digestive tract, urine from the kidneys through the ureters into the bladder, and bile from the gall bladder into the duodenum and transport of blood through the artery with mild stenosis. In addition, the mechanism of peristalsis is applied in the design of biomechanical instruments in chas heart-lung machine. Peristaltic flows are studied by assuming either small amplitude but arbitrary Reynolds number by Fung and Yih [1] Shapiro et al. [2] have presented the solutions for arbitrary amplitude and negligible inertia under long wave length approximation using a wave frame of reference. $\mathrm{Li}$ and Brasseur [3] have studied the generalized model of peristaltic transport of in a finite length tube assuming arbitrary wave shape under lubrication approach. The corresponding two dimensional channel problem has been discussed by Eytan and Elad [4] with application to intra - uterine fluid in a sagittal cross section of the uterus whose walls oscillate with either symmetrically or asymmetrically. Srivastava [5] studied the interaction of peristaltic flow with pulsatile flow when the frequency of the traveling wave and that of the imposed pressure gradient are equal. Affifi and Gad [6] investigated the interaction of Peristaltic flow with pulsatile fluid through a porous medium when the ratio between these two frequencies is equal to the wave number of the imposed pressure gradient. Acharya [7] investigated the pulsatile flow of a dusty fluid, containing small solid particles through a two dimensional constricted tube. Chaturani and Samy [8] studied the effects of non-Newtonian nature of blood and pulsatility on flow through a stenosed tube. Recently Rachid and Ouazzani [9] has obtained a solution for the problem of peristaltic pulsatile flow in a tube. Some other reported recently which are worth mentioning Ravikumar et al. [26], Rao et al. [27] and Umamaheswar et al. [28].

In many biomechanical devices such as small blood vessels, the tube wall is not rigid. The tissne region of the blood vessel is modelled as a permeable layer. In view of this it will interesting to study peristaltic pulsatile flow of a viscous fluid in a tube with permeable boundary. In the present work, we study the effect of the pulsatile flow on the peristaltic pumping of a Newtonian fluid in an axi symmetric cylindrical tube with permeable wall is investigated The analytical solution of this problem is given as on asymptotic expansion in the Womersly number $\beta$ which characterize the unsteadyness effect in the wave frame. The pumping characteristics are discussed. 


\section{Formulation Of The Problem}

Consider the peristaltic transport of an incompressible Newtonian fluid through an axisymmetric tube with permeable wall. An infinite train of sinusoidal waves travel on the wall of the tube. In a cylindrical coordinate - system $(\bar{r}, \bar{z})$ the dimensional equation for the varying tube radius is given

$$
\bar{R}=\bar{h}(\bar{z}, \bar{t})=a+b \sin \left(\frac{2 \pi}{\lambda}(\bar{z}-c \bar{t})\right)
$$

where ' $\mathrm{a}$ ' is the average radius of the tube, ' $\mathrm{b}$ ' is the amplitude of the wave, ' $\lambda$ ' is the wave length and ' $c$ ' is the wave speed.

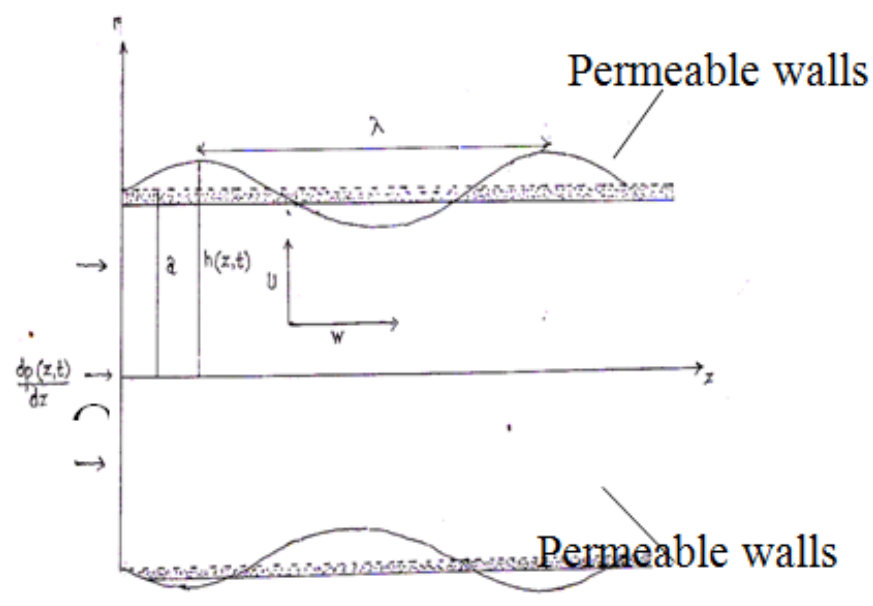

Fig: 1. Physical Model

In the absence of external forces the equations of continuity and momentum for the flow of an incompressible fluid are given by

$$
\begin{aligned}
& \operatorname{div} \bar{V}=0 \\
& \rho \cdot \frac{d \bar{v}}{d t}=-\nabla \bar{P}+\operatorname{div} \bar{\sigma}
\end{aligned}
$$

Where $\rho$ is the density, $\bar{v}$ is the velocity, $\bar{P}$ is the pressure and $\bar{\sigma}$ is the viscous stress tensor.

\section{Equations Of Motion}

The flow becomes steady in the wave frame $(\mathrm{x}, \mathrm{y})$ moving with velocity ' $c$ ' away from the fixed frame $(\mathrm{x}, \mathrm{y})$. The transformation between these two frames is given by

$$
\bar{z}=\bar{Z}-c \bar{t} \quad ; \quad \bar{r}=\bar{R}
$$

And the velocity components are also related by

$$
\bar{u}(\bar{r}, \bar{z})=\bar{U}(\bar{R}, \bar{Z}-C \bar{t}) \quad ; \quad \bar{w}(\bar{r}, \bar{z})=\bar{W}(\bar{R}, \bar{Z}-c \bar{t})-c
$$

Where $\bar{u}$ and $\bar{w}$ are the velocity components in the wave frame.

We introduce the following dimensionless variables;

$$
\begin{aligned}
& z=\frac{\bar{z}}{\lambda} ; h=\frac{\bar{h}}{a} ; r=\frac{\bar{r}}{a} ; t=\frac{\bar{t}}{T_{0}} ; u=\frac{\lambda \bar{u}}{a c} \\
& w=\frac{\bar{w}}{c} ; P=\frac{a^{2} \bar{p}}{\mu \lambda c}, Q=\frac{\bar{Q}}{2 \pi c a^{2}}
\end{aligned}
$$

In view of the continuity equation stream function $\psi(r, z)$ in dimentionless form is

$$
u(r, z)=-\frac{1}{r} \frac{\partial \Psi}{\partial z} \quad ; \quad w(r, z)=\frac{1}{r} \cdot \frac{\partial \Psi}{\partial r}
$$


The equations of motion become

$$
\begin{aligned}
& {\left[-\delta^{2} \beta \cdot \frac{\partial}{\partial t}+R_{e} \delta^{3}\left(\frac{1}{r} \frac{\partial \Psi}{\partial z} \cdot \frac{\partial}{\partial r}-\frac{1}{r} \frac{\partial \Psi}{\partial r} \cdot \frac{\partial}{\partial z}\right)\right] \cdot \frac{1}{r} \cdot \frac{\partial \Psi}{\partial z}} \\
& -\delta^{2} \frac{1}{r} \cdot \frac{\partial}{\partial r}\left(r \cdot \frac{\partial}{\partial r}\left(\frac{1}{r} \cdot \frac{\partial \Psi}{\partial r}\right)-\delta^{4} \frac{\partial}{\partial z}\left(\frac{\partial}{\partial z}\left(\frac{1}{r} \cdot \frac{\partial \Psi}{\partial z}\right)\right)\right)=\frac{-\partial p}{\partial r} \\
& {\left[\beta \cdot \frac{\partial}{\partial t}-R_{e} \delta\left(\frac{1}{r} \cdot \frac{\partial \Psi}{\partial z} \cdot \frac{\partial}{\partial r}-\frac{1}{r} \cdot \frac{\partial \Psi}{\partial r} \cdot \frac{\partial}{\partial z}\right)\right] \cdot \frac{1}{r} \frac{\partial \Psi}{\partial r}} \\
& +\frac{1}{r} \frac{\partial}{\partial r}\left(r \cdot \frac{\partial}{\partial r}\left(\frac{1}{r} \cdot \frac{\partial \Psi}{\partial r}\right)\right)+\delta^{2} \frac{\partial}{\partial z}\left(\frac{\partial}{\partial z}\left(\frac{1}{r} \cdot \frac{\partial \Psi}{\partial r}\right)\right)=\frac{-\partial p}{\partial z}
\end{aligned}
$$

Where the dimensionless wave number $\delta$, the Reynolds number $\mathrm{R}_{\mathrm{e}}$ and the Womersly number $\beta$ are defined by

$$
\delta=\frac{a}{\lambda} ; R_{e}=\frac{\rho c a}{\mu} ; \beta=\frac{\rho a^{2}}{\mu T_{0}}
$$

For $\beta=0$ the equations given by (8) and (9) coincide with those generally considered when the flow in the wave frame is steady.

Boundary conditions are

$$
\begin{aligned}
& \Psi=0, \frac{\partial}{\partial r}\left(\frac{1}{r} \cdot \frac{\partial \Psi}{\partial r}\right)=0, \text { for } \mathrm{r}=0 \\
& \Psi=F(t), \frac{1}{r} \frac{\partial \Psi}{\partial r}=-1+\frac{\sqrt{D a}}{\alpha} \cdot \frac{\partial}{\partial r}\left(\frac{1}{r} \cdot \frac{\partial \Psi}{\partial r}\right) \quad, \text { for } r=h(z)
\end{aligned}
$$

Where $D a=\frac{k}{a^{2}}, \alpha$ is the slip Parameter

$h(z)=1+\phi \operatorname{Sin}(2 \pi z)$ is the dimensionless radius of the tube

We note that (11) is the saffman (10) slip condition at the permeable boundary.

\section{Method Of Solution}

The unsteady phenomenon is due to the interaction of pulsatile flow and peristaltic transport. Assume that the flow rate $\mathrm{F}(\mathrm{t})$ in the wave frame resulting of this interaction is given by Rachid and Ouazzani, (9)

$$
F(t)=F_{0}+\beta . F_{1}(t)
$$

Where $\mathrm{F}_{0}$ is the flow rate in the wave frame in absence of the pulsatile flow and $F_{1}(t)$ is a function of time of frequency $\Omega$.

Therefore, we seek the solution of the problem under small $\beta$. We expand $\Psi$ and $P$ in the following form;

$$
\begin{aligned}
& \Psi=\Psi_{0}+\beta \Psi_{1}+\beta^{2} \Psi_{2} \\
& P=P_{0}+\beta P_{1}+\beta^{2} P_{2}
\end{aligned}
$$

Substituting these relations in the governing equation, we obtain the following Zeroth - Order system:

$$
\begin{aligned}
& \frac{\partial P_{0}}{\partial r}=0 \\
& \frac{\partial P_{0}}{\partial z}=\frac{1}{r} \cdot \frac{\partial}{\partial r}\left(r \cdot \frac{\partial}{\partial r}\left(\frac{1}{r} \cdot \frac{\partial \Psi_{0}}{\partial r}\right)\right) \\
& \Psi_{0}=0, \frac{\partial}{\partial r}\left(\frac{1}{r} \cdot \frac{\partial \Psi_{0}}{\partial r}\right)=0, \text { for } \mathrm{r}=0
\end{aligned}
$$




$$
\begin{array}{r}
\Psi_{0}=F_{0}, \frac{1}{r} \cdot \frac{\partial \Psi_{0}}{\partial r}=-1+\alpha_{0} \cdot \frac{\partial}{\partial r}\left(\frac{1}{r} \cdot \frac{\partial \Psi_{0}}{\partial r}\right) \text { at } r=h(z) \\
\text { Where } \alpha_{0}=\frac{\sqrt{D a}}{\alpha}
\end{array}
$$

\section{FIRST - ORDER SYSTEM:}

$$
\begin{aligned}
& \frac{\partial P_{1}}{\partial r}=0 \\
& \frac{\partial P_{1}}{\partial r}=\frac{1}{r} \cdot \frac{\partial}{\partial r}\left(r \cdot \frac{\partial}{\partial r}\left(\frac{1}{r} \cdot \frac{\partial \Psi_{1}}{\partial r}\right)\right) \\
& \Psi_{1}=0, \frac{\partial}{\partial r}\left(\frac{1}{r} \cdot \frac{\partial \Psi_{1}}{\partial r}\right)=0 \text { for } \mathrm{r}=0 \\
& \Psi_{1}=F_{1}(t), \frac{1}{r} \cdot \frac{\partial \Psi_{1}}{\partial r}=\alpha_{0} \frac{\partial}{\partial r}\left(\frac{1}{r} \cdot \frac{\partial \Psi_{1}}{\partial r}\right) \text { at } r=h(z)
\end{aligned}
$$

\section{SECOND ORDER SYSTEM:}

$$
\begin{aligned}
& \frac{\partial P_{2}}{\partial r}=0 \\
& \frac{\partial}{\partial t}\left(\frac{1}{r} \cdot \frac{\partial \Psi_{1}}{\partial r}\right)=-\frac{\partial P_{2}}{\partial z}+\frac{1}{r} \cdot \frac{\partial}{\partial r}\left(r \cdot \frac{\partial}{\partial r}\left(\frac{1}{r} \cdot \frac{\partial \Psi_{2}}{\partial r}\right)\right) \\
& \Psi_{2}=0, \frac{\partial}{\partial r}\left(\frac{1}{r} \cdot \frac{\partial \Psi_{2}}{\partial r}\right)=0, \text { for } \mathrm{r}=0 \\
& \Psi_{2}=0, \frac{1}{r} \cdot \frac{\partial \Psi_{2}}{\partial r}=\alpha_{0} \cdot \frac{\partial}{\partial r}\left(\frac{1}{r} \cdot \frac{\partial \Psi_{2}}{\partial r}\right) \text { at } r=h(z)
\end{aligned}
$$

\section{ZEROTH -ORDER SOLUTION:}

The solution of the problem in this order is the classical solution of steady flow

$$
\begin{aligned}
& \Psi_{0}=\frac{h\left(2 F_{0}+h^{2}\right)}{\left(4 \alpha_{0}-h\right)}\left(\frac{L^{4}}{2}-L^{2}+\frac{2 \alpha_{0}}{h} L^{2}\right)-\frac{r^{2}}{2} \\
& U_{0}=\frac{2\left(2 F_{0}+h^{2}\right)}{h\left(4 \alpha_{0}-h\right)}\left(L^{2}-1+\frac{2 \alpha_{0}}{h}\right)-1 \\
& \frac{\partial P_{0}}{\partial z}=\frac{8\left(2 F_{0}+h^{2}\right)}{h^{3}\left(4 \alpha_{0}-h\right)}
\end{aligned}
$$

\section{FIRST ORER SOLUTION:}

$$
\begin{aligned}
& \Psi_{1}=\frac{h F_{1}}{\left(4 \alpha_{0}-h\right)}\left(L^{4}-2 L^{2}+\frac{4 \alpha_{0}}{h} L^{2}\right) \\
& U_{1}=\frac{4 F_{1}}{h\left(4 \alpha_{0}-h\right)}\left(L^{2}-1+\frac{2 \alpha_{0}}{h}\right) \\
& \frac{\partial P_{1}}{\partial z}=\frac{16}{h^{4}} \frac{h F_{1}}{\left(4 \alpha_{0}-h\right)}
\end{aligned}
$$




\section{SECOND ORDER SOLUTION}

$$
\begin{gathered}
\Psi_{2}=\frac{h^{3} \frac{d F_{1}}{d t}}{\left(4 \alpha_{0}-h\right)}\left[\frac{L^{6}}{24}-\frac{L^{4}}{4}+\frac{3 L^{2}}{8}+\alpha_{0}\left(\frac{L^{4}}{2 h}-\frac{3 L^{2}}{2 h}+\frac{L^{2}}{2 h^{2}}\right)\right] \\
-\frac{4}{3 h\left(4 \alpha_{0}-h\right)}\left[h+6 \alpha_{0}\left(2 \alpha_{0}-h\right)\left(\frac{L^{4}}{8}-\frac{L^{2}}{4}+\frac{\alpha_{0} L^{2}}{2 h}\right)\right] \\
U_{2}=\frac{h \cdot \frac{d F_{1}}{d t}}{\left(4 \alpha_{0}-h\right)}\left[\frac{L^{4}}{4}-L^{2}+\frac{3}{4}+\alpha_{0}\left(\frac{2 L^{2}}{h}+\frac{\alpha_{0}}{h^{2}}-\frac{3}{h}\right)\right] \\
\frac{4}{3\left(4 \alpha_{0}-h\right)}\left(h^{2}+6 \alpha_{0}\left(2 \alpha_{0}-h\right)\right)\left(\frac{L^{2}}{2 h}-\frac{1}{2 h}+\frac{\alpha_{0}}{h^{2}}\right) \\
\frac{\partial P_{2}}{\partial z}=-8 \cdot \frac{d F_{1}}{d t} \frac{h^{2}+6 \alpha_{0}\left(2 \alpha_{0}-h\right)}{3 h^{2}\left(4 \alpha_{0}-h\right)^{2}}
\end{gathered}
$$

Summing up, we write the perturbation solution as follows

$$
\begin{aligned}
& \Psi=\frac{h}{\left(4 \alpha_{0}-h\right)}\left[\left(2 F+h^{2}\right)\left(L^{4}-2 L^{2}+\frac{4 \alpha_{0}}{h} L^{2}\right)-\frac{r^{2}}{2}+\beta \cdot h^{2} \cdot \frac{d F}{d t}\left(\frac{L^{4}}{24}-\frac{L^{2}}{4}+\frac{3 L^{2}}{8}\right)\right] \\
& +\alpha_{0}\left(\frac{L^{4}}{2 h}-\frac{3 L^{2}}{2 h}+\frac{L^{2}}{2 h^{2}}\right)-\frac{4}{3 h\left(4 \alpha_{0}-h\right)}\left(h+\alpha_{0}\left(2 \alpha_{0}-h\right)\left(\frac{L^{4}}{8}-\frac{L^{2}}{4}+\frac{\alpha_{0} L^{2}}{2 h}\right)\right) \\
& U=\frac{4}{\left(4 \alpha_{0}-h\right)}\left[\left(2 F+h^{2}\right)\left(L^{2}-1+\frac{2 \alpha_{0}}{h}\right)-1+\beta \cdot h \cdot \frac{d F}{d t}\left(\frac{L^{4}}{4}-L^{2}+\alpha_{0}\left(\frac{2 L^{2}}{4}+\frac{\alpha_{0}}{h^{2}}-\frac{3}{h}\right)\right)\right] \\
& -\frac{4}{3\left(4 \alpha_{0}-h\right)}\left[h^{2}+6 \alpha_{0}\left(2 \alpha_{0}-h\right)\left(\frac{L^{2}}{2 h}-\frac{1}{2 h}+\frac{\alpha_{0}}{h^{2}}\right)\right] \\
& \frac{\partial P}{\partial z}=\frac{8}{h^{3}\left(4 \alpha_{0}-h\right)}\left[\left(2 F+h^{2}\right)-\beta \cdot \frac{d F}{d t} \frac{h^{2}+6 \alpha_{0}\left(2 \alpha_{0}-h\right) h}{3\left(4 \alpha_{0}-h\right)}\right]
\end{aligned}
$$

We observe that equation (27)-(32) reduce to the corresponding results of Rachid and Ouazzani (9) for pulsatile peristaltic flow in an impermeable flexible tube.

The volume rate of flow in the fixed $\bar{R}, \bar{Z}$ coordinate system is given as

$$
\bar{Q}(\bar{z}, \bar{t})=2 \pi \int_{0}^{\bar{h}} \bar{W}(\bar{z}, \bar{t}) \bar{R} d \bar{R}
$$

On substituting (25) and (26) into (33) and integrating we obtain

$$
\bar{Q}(\bar{z}, \bar{t})=\bar{q}+\pi c \bar{h}^{2}
$$

Where

$$
\bar{q}=2 \pi \int_{0}^{h} \bar{w} \cdot \bar{r} \cdot d \bar{r}
$$

Is the volume flow rate in the moving coordinate system.

The space - mean flow over a wave length is defined by

$$
\bar{Q}(\bar{t})=\frac{1}{\lambda} \int_{0}^{\lambda} \bar{Q} d \bar{z}
$$


Using dimensionless variables, we write

$$
\frac{\bar{Q}(\bar{t})}{2 \pi c a^{2}}=\frac{\bar{q}(\bar{t})}{2 \pi c a^{2}}+\frac{1}{2}\left(1+\frac{\phi^{2}}{2}\right)
$$

The equation (37) becomes

$$
\theta(t)=F(t)+\frac{1}{2}\left(1+\frac{\phi^{2}}{2}\right)
$$

With

$$
\theta(t)=\frac{\bar{Q}(\bar{t})}{2 \pi c a^{2}} \text { and } F(t)=\theta(t)-\frac{1}{2}\left(1+\frac{\phi^{2}}{2}\right)
$$

Where $\theta(t)$ and $F(t)$ are respectively the flow rates in the fixed and the wave frames.

Integrating the equation (32) with respect to $\mathrm{z}$ over one wave length we get the pressure rise over one cycle of the wave as

$$
\begin{aligned}
& \Delta P=-\int_{0}^{1} \frac{\partial p}{\partial z} \cdot d z \\
& \Delta P=-\int_{0}^{1} \frac{8}{h^{3}\left(4 \alpha_{0}-h\right)}\left\{2\left(\theta(t)-\frac{1}{2}\left(1+\phi^{2} / 2\right)+h^{2}\right)\right. \\
& \left.-\beta\left(\frac{d\left(\theta(t)-\frac{1}{2}\left(1+\phi^{2} / 2\right)\right)}{d t} \cdot \frac{h^{2}+6 \alpha_{0}\left(2 \alpha_{0}-h\right) h}{3\left(4 \alpha_{0}-h\right)}\right)\right\} d z
\end{aligned}
$$

The dimensionless friction force $\mathrm{F}$ at the wall across one wave length in given by

$$
F=\int_{0}^{1} h^{2}\left(-\frac{\partial p}{\partial z}\right) \cdot d z
$$

\section{Discussion Of Results}

We have calculated the pressure difference from equation (40) as a function of $\theta(t)$ for different values of amplitude ratio $\phi$ with fixed Darcy number $D a=0.0001$ and slip parameter $\alpha=0.5$ and is shown in Fig(2). We observe that $\Delta p$ the larger the amplitude ratio, the greater the pressure rise against which the pump works. For free pumping there is an increase in flux with increasing amplitude ratio. The Variation of the pressure difference with $\theta(t)$ for different values of Darcy number Da with fixed amplitude ratio $\phi=0.4$ and slip parameter $\alpha=0.5$ is evaluated from equation (40) and is shown in Fig(3). We observe that $\Delta p$ increases with increasing Darcy number for a given $\theta$. We calculate the pressure difference form equation (40) as a function of $\theta(t)$ for different values of slip parameter $\alpha$ with fixed amplitude ratio $\phi=0.4$ and Darcy number $D a=0.0001$ and is shown in Fig(4). We observe that $\Delta p$ decreases with increasing slip parameter $\alpha$ for a given $\theta$. We have calculated the frictional force $\mathrm{F}$ from equation (40) as function of $\theta$ for different values of amplitude ratio $\phi$ with fixed $D a=0.0001$ and $\alpha=0.5$ and is shown in Fig(5). We observe that the frictional force $\mathrm{F}$ has is opposite behaviour when compared to pressure rise $\Delta p$. We have calculated the frictional force $\mathrm{F}$ as function of $\theta$ for different values of Darcy number Da with fixed amplitude ratio $\phi=0.4$ and slip parameter $\alpha=0.5$ from equation (41) and is shown in Fig(6). We observed 
that $\mathrm{F}$ exhibts opposite behaviour when compared to pressure rise $\Delta p$. The variation of the frictional force $\mathrm{F}$ as function of $\theta$ is evaluated form equation (41) for different values of slip parameter $\alpha$ with fixed amplitude ratio $\phi=0.4$ and Darcy number $D a=0.0001$ and is shown in Fig(7). We notice that F shows opposite behaviour compared to pressure rise $\Delta p$

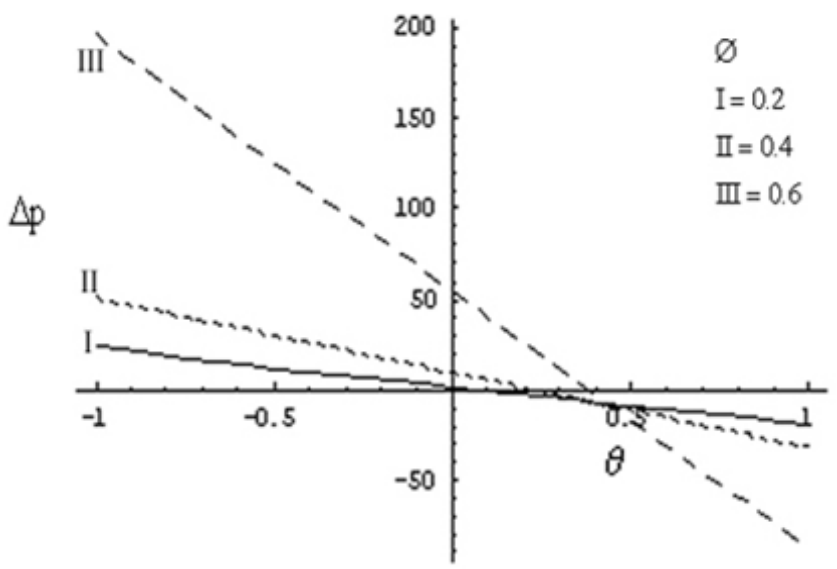

Fig .2. The variation of $\sqcup \mathrm{p}_{\text {with }} \Phi$ for fixed $\mathrm{Da}=0.0001, \alpha=0.5$ for different $\Phi$ values

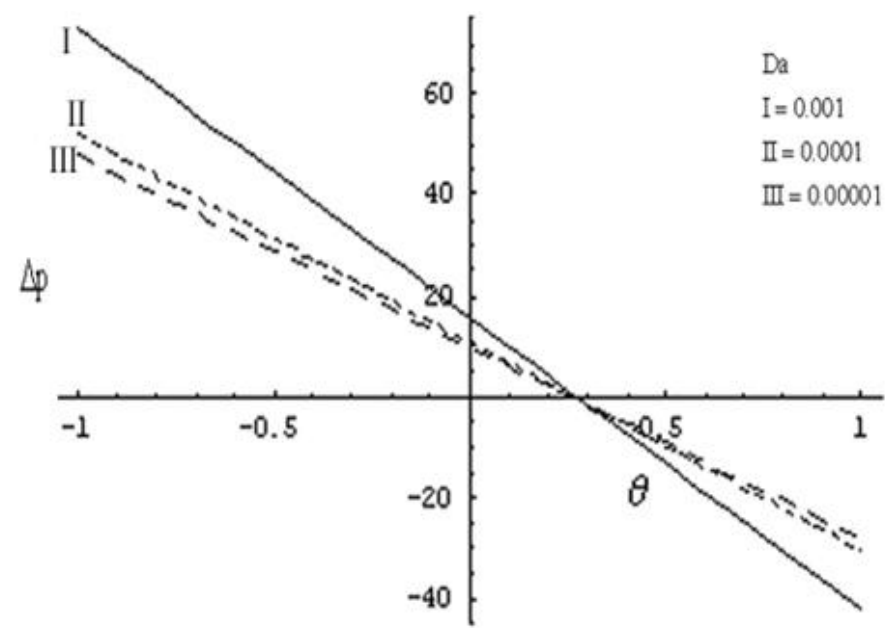

Fig .3. The variation of $\square \mathrm{p}$ with $\Phi$ for fixed $\Phi=0.4, \alpha=0.5$ for different $D a$ values

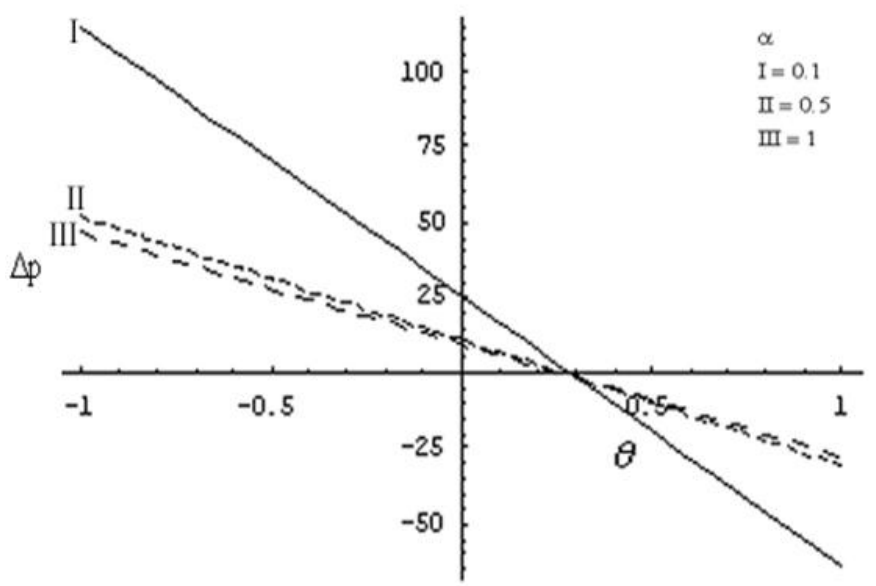

Fig .4. The variation of $\sqcup p_{\text {with }} \Phi$ for fixed $\Phi=0.4, \mathrm{Da}=0.0001$ for different $\alpha$ values 


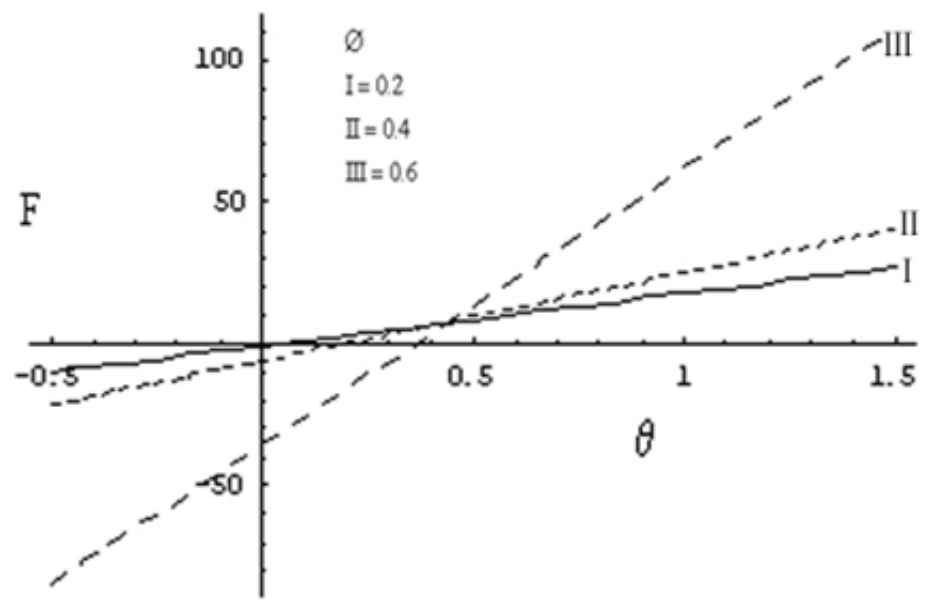

Fig.5.The variation of $\mathrm{F}$ with $\Phi$ for fixed $\mathrm{Da}=0.0001, \alpha=0.5$ for different $\Phi$ values

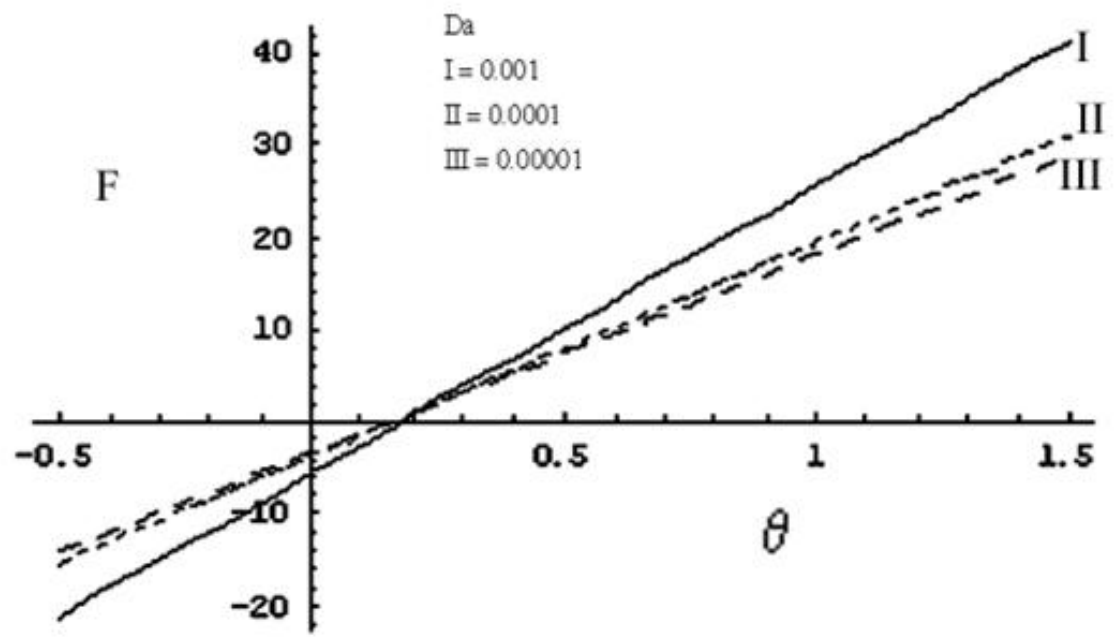

Fig.6.The variation of $\mathrm{F}$ with $\Phi$ for fixed $\Phi=0.4, \alpha=0.5$ for different $\mathrm{Da}$ values

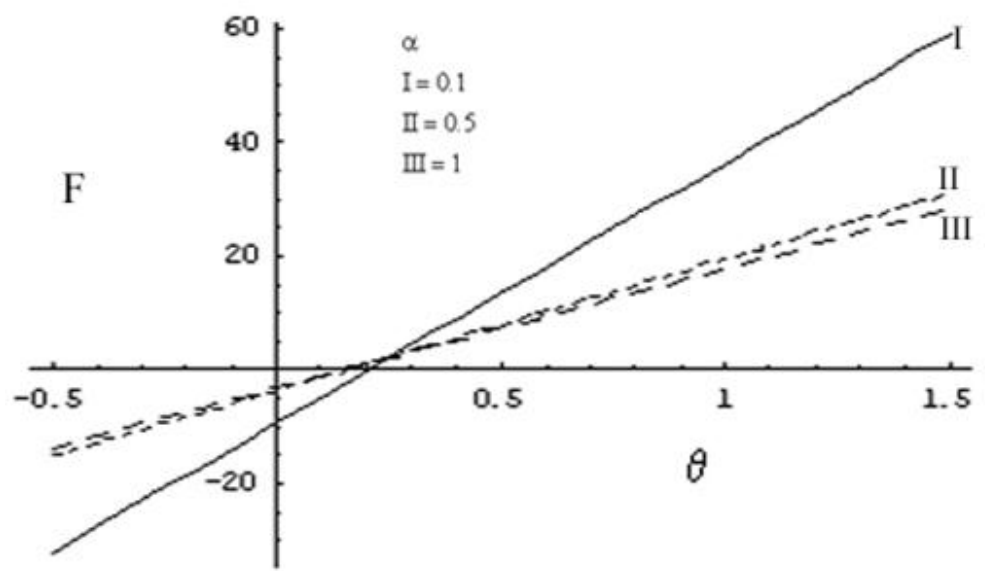

Fig.7.The variation of $\mathrm{F}$ with $\Phi$ for fixed $\Phi=0.4, \mathrm{Da}=0.0001$ for different $\alpha$ values

\section{References}

[1]. Fung and Yih, Maximum flow rate versus Reynolds number for small amplitude ratios. J. Applied Mech, 35, 669 - 675, (1968).

[2]. A.H. Shapiro, M. Y. Jaffrin and S. L. Weinberg, Peristaltic pumping with long wavelengths at low Reynolds number. J. fluid Mech, $37,799-825,(1969)$.

[3]. Li and Brasseur, Non-Study Peristaltic transport in finite length tubes. J.fluid Mech, 248, 129-151, (1993).

[4]. Eytan and D.Elad, Analysis of intra - uterine fluid motion induced by uterine contractions. J. Bulletin of Mathematical Biology, 61, 221- 238,(1999). 
The Effect of Pulsatile Flow on The Peristaltic Transport of A Newtonian Fluid In A Cylindrical ..

[5]. Srivastava, M. Interaction of peristaltic flow with pulsatile flow in a circular cylindrical tube. Biomechanics,, 18(4), 247 - 253, (1985).

[6]. Afifi, N. A. S., and N. S. Gad, Interaction of peristaltic flow with pul-satile fluid throngh a porous medium, Applied Mathematics and Compu-tation, 142, $167-176$, (2003).

[7]. Acharya, G. R. K. Pulsatile flow of a Dusty fluid through a constricted channel, J. of Applied Math. Phys. (ZAMP), 29 (1981).

[8]. Chaturani, P., and R. P. Samy. Pulsatile flow of Casson's fluid through stenosed arteries with applications to blood flow, Biorheology, 23(5), 499- 511, (1986).

[9]. Rachid and M. T.Ouazzani, The effect of a pulsatile flow on the peristaltic out put case of a Newtonian fluid. Adv. Studies Theor. 2, 291-307,(1971).

[10]. Saffman, P.G Appl. Math., Vol. 20, P. 93, (1971).

[11]. Brasseur, J.G., Corrsin. S. and Lu, N.Q. The influence of a peripheral layer of different viscosity on peristaltic pumping with Newtonian fluid. J, Fluid. Mech. 174, 495 - 519, (1987).

[12]. Burns, J.C and Parkes, T. Peristaltic motion. J. Fluid Mech. 29, 731 - 743, (1967).

[13]. Barton, C., and S. Raynor, Peristaltic Flow in Tubes, Bull. Math. Biophys, 30, 663 - 680, (1968).

[14]. Jaffrin, M, Y. and Shapiro, A.H. Peristaltic pumping. Ann. Rev. Fluid. Mech. 3, 13 - 36, (1971).

[15]. Mishra, M and A. Ramachandra Rao, Peristaltic transport of a Newtonian fluid in an asymmetric channel, Z. angew. Math. Phys, $54.532-550,(2003)$.

[16]. Jaffrin, M. Y., and A.H. Shapiro, Peristaltic pumping. Ann. Rev. Fluid Dyn, 3, 13-37, (1971).

[17]. Manton M. J. Manton, Long-wavelength peristaltic pumping at low Reynolds number J. Fluid Mech, 68, 467 - 476, (1975).

[18]. Mittra, T.K. and Prasad, S.N. On the influence of wall properties and Poiseuille flow in the peristalsis. J. Biomechanics, p: 681 693, (1973).

[19]. Afifi, N. A. S., and N. S. Gad. Interaction of peristaltic flow with pulsatile magneto-fluid through a porous medium, Ada Mechanica, 149, 229 - 237, (2001).

[20]. Rao, A. R and M. Mishra, Nonlinear and curvature effects on peristaltic flow of a viscous fluid in an asymmetric channel, Acta Mechan-ica, 168, 35- 59, (2004).

[21]. Rao, A.R. and Usha S. Peristaltic transport of the immiscible viscous fluids in a circular tube. J. Fluid Mech. 298, 271 - 285, (1995).

[22]. Subba Reddy. M.V., Manoranjan Mishra, Sreenadh S. and Ramachandra Rao. A. Influence of lateral walls on peristaltic. Flow in a rectangular duct, Transactions of the ASME. J. Fluids Engg. 127 (2005).

[23]. Vajravelu, K., Sreenadh, S and Ramesh Babu, V. Peristaltic transport of a Hershel - Bulkely fluid in an inclined tube, Inf J. Non linear Mech 40, 83 - 90, (2005a).

[24]. Vajravelu, K., Sreenadh, S., and Ramesh Babu, V. Peristaltic transport of a Hershel - Bulkely fluid in a channel, Appl. Mech. And comput., 169, $726-735,(2005$ b).

[25]. Ravikumar V, Raju M.C, Raju G.S.S.,MHD three dimensional Couette flow past a porous plate with heat transfer, IOSR Jour.Maths., Vol. 1, no.3,pp.3-9,2010.

[26]. Rao, B. M., Reddy, G. V. R., M.C.Raju., S.V.K,Varma, MHD transient free convection and chemically reactive flow past a porous vertical plate with radiation and temperature gradient dependent heat source in slip flow regime, IOSR Journal of applied physics, Vol. 3(6), 2013,22-32.

[27]. Umamaheswar, M., S.V.K.Varma, M.C.Raju, Numerical study of Magneto-Convective and radiation abosorption fluid flow past an exponentially accelerated vertical porous plate with variable temperature and concentration in the presence of Soret and Dufour effects, IOSR Journal of Mathematics, Volume12, and Issue2 Ver. I (Mar.-Apr.2016), pp 109-120. DOI:10.9790/5728-1221109120. 